\title{
Patrones de personalidad y asertividad sexual en agresores sexuales recluidos en cuatro centros penitenciarios de Perú
}

\section{Personality patterns and sexual assertiveness in sexual aggressors confined in four Peruvian penitentiaries}

\author{
Sixto Serafín Bonifacio Gutiérrez* \\ Escuela Profesional de Psicología, \\ Universidad de San Martín de Porres, Perú
}

\section{Resumen}

Se determina la relación entre los patrones de personalidad y asertividad sexual en reclusos que cumplen condena por delito de agresión sexual, en cuatro centros penitenciarios de Perú. El tipo de estudio es cuantitativo, de diseño descriptivo correlacional transversal, en el que participaron 184 reclusos, cuyas edades fluctuaron entre 18 y 60 años. En la recolección de datos, se utilizó el Inventario Clínico Multiaxial de Millon II (MCMI-II) y la Escala de Asertividad Sexual (SAS). Los resultados evidencian que existe una relación significativa y negativa entre las variables de estudio. Además, se encontró que 11 de 13 patrones de personalidad tienen una relación significativa y negativa con un nivel bajo de asertividad sexual; con mayor acentuación en las dimensiones de inicio y rechazo.

Palabras clave: patrones de personalidad; asertividad sexual; agresión sexual

\section{Abstract}

The present article aims to determine the relationship between personality patterns and sexual assertiveness in inmates serving sentences for sexual

Este es un artículo Open Access bajo la licencia Creative Commons Atribución-NoComercial-Compartirlgual 4.0

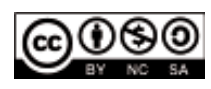


aggression in four Peruvian prisons. This study had a quantitative, descriptive, correlational and cross-sectional design, and consisted of 184 inmates aged between 18 and 60. The Millon Clinical Multiaxial Inventory II (MCMI-II) and the Sexual Assertiveness Scale (SAS) were used for data collection. The results showed that there is a significant and negative relationship among the study variables. It was found that 11 out of 13 personality patterns had a significant and negative relationship, showing a low level of sexual assertiveness and more emphasis on the dimensions of onset and rejection of sexual activity.

Keywords: personality patterns; sexual assertiveness; sexual aggression

\section{Introducción}

La incidencia actual sobre la violencia sexual es alarmante en el país y en el mundo. Nos encontramos frente a un grave problema psicosocial que afecta a muchos hogares y a la sociedad en general. Por ello, los profesionales de la salud mental tenemos como deber comprender, explicar y plantear alternativas de solución frente a este problema que padecen niños, adolescentes y adultos.

Para comprender la conducta sexual agresiva, hay que abordarla desde diferentes niveles: personal, psicológico, familiar, cultural y social.

En este estudio se pretende establecer la relación entre la personalidad del agresor sexual y los componentes de la asertividad sexual, con el objetivo de hallar explicaciones respecto del correlato de estas dos variables, lo que a su vez, permita plantear medidas correctivas mediante nuevas propuestas de intervención en la prevención y el tratamiento del problema de la violencia sexual.

\section{Aspectos teóricos}

\section{Personalidad}

La personalidad es un constructo que reúne tres grandes dimensiones: biológica, psicológica y social; de la relación estrecha entre estas tres 
dimensiones o componentes se estructura la identidad de sí mismo, sus necesidades y la motivación que impulsa su desarrollo.

Millon (2006) define la personalidad como «un patrón complejo de características psicológicas profundamente enraizadas, que se expresan de forma automática en casi todas las áreas de la actividad psicológica» (p. 2). Este patrón reúne componentes cognitivos, afectivos y conductuales que forman parte del repertorio conductual por largos periodos de tiempo, siendo producto tanto de disposiciones orgánicas, como del aprendizaje experimental. Esto es, en la formación de la personalidad de un individuo, se considera cómo interactúa este frente a las demandas del medio ambiente y cómo se relaciona consigo mismo.

Las definiciones de personalidad se desprenden de diferentes teorías, la coincidencia en todas ellas radica en atribuirle a su estructura factores internos, guiados por impulsos biológicos y factores externos que tienen relación con el carácter y el aprendizaje en la interacción con el medio social a lo largo de la vida.

\section{Definición de los trastornos de personalidad}

De acuerdo a la Asociación Americana de Psiquiatría (2014), se reconoce un trastorno de personalidad por la presencia de características específicas y permanentes, que difieren de lo establecido como «normal» para la cultura del individuo. Estos rasgos se manifiestan a nivel interno y en la conducta del sujeto, acompañan a la persona en distintas esferas de su vida, son patrones inflexibles, que permanecen estables en el tiempo. Generalmente, se inician en la adolescencia o en la edad adulta temprana y afectan el normal desempeño del sujeto.

Dicho de otra forma, los trastornos de personalidad se caracterizan por afectar la forma en que las personas perciben, reaccionan y se relacionan con los estímulos de su entorno (Asociación Americana de Psiquiatría, 2014). Estos rasgos presentan cierta estabilidad en el tiempo, se determinan por ser inalterables y por responder de diferente modo a lo socialmente aceptado. Así, las personas que presentan un trastorno de personalidad pueden responder a su entorno de acuerdo a características específicas, 
interiorizadas, tomadas como habituales para su particular configuración del mundo, pero que a su vez, se asemejan a los rasgos que otra persona podría presentar.

Una persona que presenta un trastorno de personalidad ve disminuida su capacidad para relacionarse con su entorno, ya que permanece en constante lucha por adaptarse, pese a la rigidez en su accionar y en su pensamiento, probablemente a lo largo de toda su vida. Esta rigidez hace que el sujeto sea proclive a afectar su normal desenvolvimiento en diversas áreas de desempeño: personal, familiar, social, laboral, relación de pareja, entre otros; experimentando niveles altos de insatisfacción, lo cual hace que sus relaciones interpersonales sean conflictivas. La persona no es consciente de lo inapropiado que resultan sus pensamientos o conductas para los demás, tomándolos más bien como regulares o apropiados.

Las clasificaciones de los trastornos de personalidad que plantean la Clasificación Estadística Internacional de los Trastornos Mentales y del Comportamiento, décima revisión (CIE-10) y el Manual Diagnóstico y Estadístico de los Trastornos Mentales, quinta edición (DSM-5), nos ayudan a identificar un prototipo de estructura alterada que se van desarrollando a lo largo de la vida, estas estructuras patológicas no son tan fáciles de determinarlas, hay que hacer uso de criterios clínicos rigurosos y fundamentados en el accionar de la personas para identificarlos como tales (ver Tabla 1).

\section{Asertividad sexual}

Morokoff et al. (1997) la define como «la capacidad de las personas para iniciar la actividad sexual, rechazar la actividad sexual no deseada y negociar el empleo de métodos anticonceptivos de barrera (condón), para asumir comportamientos sexuales saludables» (como se cito en TorresObregón, Onofre-Rodríguez, Sierra, Benavides-Torres, \& Garza-Elizondo, 2017, p. 35). Es necesario deslindar similitudes entre este término y asertividad per sé, ya que, pese a compartir características en cuanto a toma de decisiones, ser asertivo sexualmente está intrínsecamente relacionado con la actividad sexual. 
Tabla 1

Clasificación de los trastornos de personalidad

\begin{tabular}{ll}
\hline \multicolumn{1}{c}{ DSM-5 } & \multicolumn{1}{c}{ CIE-10 } \\
\hline Grupo A: & Trastorno paranoide de la personalidad \\
Trastorno de la personalidad paranoide & $\begin{array}{l}\text { Trastorno esquizoide de la } \\
\text { personalidad }\end{array}$ \\
$\begin{array}{l}\text { Trastorno de la personalidad } \\
\text { esquizoide }\end{array}$ & Trastorno disocial de la personalidad \\
Trastorno de la personalidad & Trastorno de inestabilidad emocional \\
esquizotípica & de la personalidad \\
Grupo B: & Trastorno histriónico de la personalidad \\
Trastorno de la personalidad antisocial & Trastorno anancástico de la \\
Trastorno de la personalidad límite & personalidad \\
Trastorno de la personalidad histriónica & Trastorno ansioso o por evitación de la \\
Trastorno de la personalidad narcisista & personalidad \\
Grupo C: & Trastorno dependiente de la \\
Trastorno de la personalidad evasiva & personalidad \\
Trastorno de la personalidad & $\begin{array}{l}\text { Otros trastornos de personalidad } \\
\text { dependiente }\end{array}$ \\
Trastorno de la personalidad obsesivo- & Trastorno de personalidad, sin \\
compulsiva & especificar \\
\hline
\end{tabular}

Nota: Adaptado de la Asociación Americana de Psiquiatría (2014).

Por ello y por otros factores psicosociales (Santos-Iglesias \& Sierra, 2010), en diversas investigaciones se afirma la dificultad que presentan las mujeres para desarrollar asertividad sexual en comparación con los varones, siendo estos últimos, quienes presentan mayor nivel, independientemente de sus conductas frente a la sexualidad y a temas relacionados con esta. Entonces, la asertividad sexual comprende las habilidades o recursos que tienen las personas para interactuar adecuadamente en las relaciones sexuales con las parejas, respetando sus derechos, actuando con madurez y respeto.

\section{Factores asociados a la asertividad sexual}

Zerubavel y Messman-Moore (2013) buscaron identificar aquellos factores relacionados a la asertividad sexual, logrando identificar dos grandes 
barreras: por un lado, la sensación de incapacidad o incompetencia frente a situaciones que involucren hablar de sexo o sexualidad y, por otro lado, ciertos factores cognitivos relacionados con la autorregulación emocional, tales como la dificultad para aceptar sus propias emociones, la falta de conciencia emocional, entre otros.

Santos-Iglesias y Sierra (2010) abordaron también dicha tarea, mediante el análisis de las correlaciones de la asertividad sexual y otros factores encontrados en diversas investigaciones, dilucidando que este constructo guarda relación con características demográficas, culturales, experienciales en cuanto al sexo y conductas psicosexuales en la población.

\section{El agresor sexual}

La Organización Mundial de la Salud (OMS) define la violencia sexual:

Como todo acto sexual, la tentativa de consumar un acto sexual, insinuaciones no deseadas, las acciones para comercializar o utilizar de cualquier otro modo la sexualidad de una persona mediante coacción por otra persona, independientemente de la relación de esta con la víctima, en cualquier ámbito, incluidos el hogar y el lugar de trabajo. (como se citó en Organización Panamericana de la Salud, 2013, p. 2)

Asimismo, la violencia sexual destaca la participación no consensuada de uno de los implicados en un acto de índole sexual. Este hecho violento es realizado de manera intencional por el agresor y atenta contra la integridad emocional y física del agredido. Además, la literatura sobre el tema manifiesta que cualquier tipo de violencia de carácter sexual, realizada en contra de otro individuo y que atente contra su libertad sexual, puede ser considerada una agresión sexual, aunque esta no se encuentre asociada directamente al delito de violación.

Según Vásquez (2005) diversas investigaciones sostienen la inexistencia de un perfil de la personalidad de un agresor sexual, sin embargo, destacan características personales en ellos, tales como una «conducta general violenta, menor autoconcepto, déficit asertivo, [...] autoritarismo, sistema 
de creencias y escasas habilidades para el manejo del estrés» (Soria \& Hernández, 1994, p. 99). Aunque evidentemente debido a la cobertura de diversos casos en los medios de comunicación, son varones agresores los que constituyen un gran porcentaje del total, también son mujeres agresoras quienes conforman una cifra nada desestimable. Como expresan Larrotta y Rangel-Noriega (2013), la explicación a la mayor incidencia de casos donde el varón es agresor podría residir en que este legitima desde pequeño conductas relacionadas con el abuso. Aunque estos autores no definen un perfil del agresor, queda delimitada a grandes rasgos la edad de la mayoría de agresores: edades fluctuantes entre la adultez temprana y media, no obstante, este dato no es predictor suficiente para tipificar a una persona como agresor.

El agresor sexual tiene todas las características antes señaladas pero el problema está en que no son perceptibles externamente de manera cotidiana, ya que no están dirigidas a este fin. Sobrellevan dichas características como impulsos internos que hace presión hasta que en un momento rebasa los controles y sale en situaciones propicias para él y cuando esto se exterioriza, utiliza la manipulación atemorizando a sus víctimas para mantener oculto el acto.

Aunque sin establecer un patrón de personalidad definido para un abusador sexual, De la Torre (2012) reconoce rasgos dependientes, antisociales, compulsivos, etc. en agresores sexuales; así como comportamientos antisociales, reacciones hostiles, ira e impulsividad en niveles superiores al promedio, baja autoestima y dificultad para establecer empatía con sus semejantes. En términos generales, el agresor sexual presenta dificultad para equilibrar sus emociones y afectos, lo cual termina reflejándose en una conducta mucho más inhibitoria y reservada, que repercute también en su conducta sexual. Esta pasividad en su accionar termina por limitar la interacción con sus pares, afectando también su búsqueda por el afecto de otra persona. Dicha restricción es de gran importancia para entender cómo en respuesta a esta situación, el individuo rechaza su conducta sexual, como una forma de protegerse a sí mismo.

En cuanto a la personalidad de los agresores sexuales, se ha encontrado que poseen un bajo nivel de asertividad sexual, que muchos de los jóvenes que 
cometen abuso sexual tienen dificultades de autoestima, falta de confianza, habilidades mínimas asertivas (Ramírez, 2002). Jiménez (2009) reporta en la personalidad de los agresores sexuales el impedimento para establecer una comunicación asertiva con las mujeres, una disminución en el control de la libido, disminución en las habilidades sociales, además lo describe con fuertes sentimientos de inseguridad asociado a la incapacidad para relacionarse de manera genuina y comprometida con los demás, lo que implica falta de empatía.

Marshall (como se citó en Soria \& Hernández, 1994, p. 27) sostiene que en estas personalidades se ha encontrado que $50 \%$ de violadores y $45 \%$ de abusadores de infantiles muestran déficit en la asertividad sexual, específicamente para establecer relaciones íntimas, lo que los ha conducido a la soledad y a una disposición agresiva manifestada en proponer relaciones sexuales bajo amenaza.

Por consiguiente, se plantea que la asertividad sexual tiene una relación altamente significativa con la personalidad del agresor sexual; centrada básicamente en la inseguridad, inmadurez, déficit en las habilidades sociales, descontrol de los impulsos, falta de empatía y sentimientos de frustración en el área sexual, lo cual potencia su agresión hacia sus víctimas. Dicha premisa es la que sustenta el propósito del presente trabajo.

\section{Método}

\section{Diseño}

La investigación se caracteriza por ser de tipo cuantitativa y tener un diseño descriptivo correlacional (Sánchez, Reyes, \& Mejía, 2018) que permite describir las relaciones entre los patrones de personalidad y el nivel de asertividad sexual en internos de centros penitenciarios por el delito de agresión sexual.

\section{Participantes}

La población la integraron internos por delitos contra el pudor y violencia sexual en establecimientos penitenciarios del Perú, de los departamentos 
de San Martín, Puno, Ancash y Lima, en el año 2017, con edades comprendidas entre los 18 y 60 años, procedentes de diferentes zonas del país; todos ellos privados de su libertad por el delito de violencia o agresión sexual en mujeres menores y adultas.

El método de muestreo es no probabilístico de tipo intencional, participaron un total de 184 internos de cuatro centros penitenciarios; 30 de Juliaca; 17 de Chimbote, 33 de Tarapoto, y 104 de Lima.

Para la selección de los participantes, se tomaron en cuenta los siguientes criterios de inclusión: tener una edad comprendida entre los 18 y 60 años, ser varones, estar sentenciados por delito de agresión sexual, estar recluidos en un penal, estar conscientes de sus actos y de su realidad circundante y participar voluntariamente del estudio.

Como criterios de exclusión se consideró: presentar datos demográficos incompletos en las pruebas, haber omitido responder más del 10\% de preguntas en la prueba y presentar puntuaciones altas en las escalas de validez, sinceridad, deseabilidad social, autodescalificación y/o alteraciones en el instrumento (MCMI-II).

\section{Instrumentos}

- Sexual Assertiveness Scale (SAS). Esta versión fue derivada de la original planteada por Morokoff et al. en 1997, siendo adaptada al español por Sierra, Vallejo-Medina y Santos-Iglesias en el año 2011. Esta escala considera tres componentes del constructo: inicio de relaciones sexuales deseadas, rechazo de las relaciones no deseadas y la prevención de embarazos y enfermedades de transmisión sexual. Puede ser administrada de forma individual o colectiva, tanto a nivel global como individual. La escala SAS está integrada por 18 ítems, los cuales se puntúan a través de una escala de respuesta tipo Likert que oscila entre 0 (Nunca) y 4 (Siempre). La mitad de los ítems están redactados de forma inversa $(3,4,6,8,10,11,13,14$ y 16), siendo las puntuaciones altas aquellas que indican mayor asertividad sexual. Para el presente estudio, la escala pasó por criterio de jueces, de donde se obtuvo baremos utilizando el método de percentiles, considerando dos cortes: 25 y 75, para ubicar las puntuaciones en tres niveles: bajo, moderado y alto. 
En la evaluación de las propiedades psicométricas del instrumento se encontró que la versión original de la Escala de Asertividad Sexual (SAS) de Morokoff et al. (como se citó en Torres-Obregon et al., 2017, p. 35) reportó buena fiabilidad (Inicio $\alpha=.77$; Rechazo $\alpha=.74$; E-ETS $\alpha=.82 \mathrm{y}$ escala global $\alpha=.82)$ y validez interna (RMSR $=.04)$ y externa $(r=.65$, $p<.001)$. Asimismo, la adaptación española de la prueba, llevada a cabo por Sierra, Vallejo-Medina y Santos-Iglesias (2011), demostró también buenas propiedades psicométricas. Se realizó un análisis factorial exploratorio, aislando las tres dimensiones del modelo original de Morokoff et al., y la estructura fue confirmada después mediante análisis factorial confirmatorio, hallándose valores que oscilaban entre un alfa de Cronbach de .76 y .85 en cuanto a su fiabilidad.

En cuanto a las puntuaciones de los componentes del instrumento y a la información recopilada durante la investigación se elaboró la Tabla 2 que muestra la distribución de las mismas.

Tabla 2

Distribución de las puntuaciones de los componentes del SAS

\begin{tabular}{lcccc}
\hline & $\begin{array}{c}\text { Puntaje } \\
\text { global }\end{array}$ & $\begin{array}{c}\text { Inicio de la } \\
\text { actividad sexual }\end{array}$ & $\begin{array}{c}\text { Rechazo de la } \\
\text { actividad sexual }\end{array}$ & $\begin{array}{c}\text { Prevención de } \\
\text { ETS }\end{array}$ \\
\hline Bajo & $0-32$ & $0-10$ & $0-10$ & $0-9$ \\
Moderado & $33-39$ & $11-13$ & $11-13$ & $10-13$ \\
Alto & 40 a más & 14 a más & 14 a más & 14 a más \\
\hline
\end{tabular}

Nota: Elaboración propia.

- Inventario Clínico Multiaxial de Millon II (MCMI-II). La versión original de la prueba fue elaborada por Theodore Millon en el año 1999, con el objetivo de evaluar los prototipos de personalidad. Este instrumento está compuesto por un manual de 175 preguntas, una hoja de respuestas, una hoja de resultados y una hoja de gráfico, en donde se puede apreciar el nivel de las diversas escalas. Los ítems de la prueba son de tipo verbal y dicotómico. Está constituida por 4 escalas de validez (validez, sinceridad, deseabilidad social, autodescalificación), 10 escalas de posturas clínicas de personalidad (esquizoide, evitativo, dependiente, histriónico, narcisista, antisocial, agresivo-sádico, compulsivo, pasivo-agresivo, autoderrotista); 3 
escalas de patología severa de personalidad (esquizotípico, borderline y paranoide); 6 escalas de síndromes clínicos (ansiedad, somatomorfo, bipolar, distimia, dependencia al alcohol, dependencia a drogas) y, por último, 3 escalas de síndromes severos (desorden del pensamiento, depresión mayor y desorden delusional). Para el presente estudio se tomó una muestra piloto, compuesta por 20 internos del penal de Tarapoto, donde se modificó la redacción de 20 ítems $(6,19,28,38,46$, 48, 51, 55, 69, 70, 81, 115, 118, 121, $123,144,160,167,168,174)$.

En cuanto a los índices de confiabilidad de la versión original en inglés del MCMI II, los índices de consistencia interna fueron bastante significativos fluctuando entre .81 (Escala PP: Trastorno delirante) y .95 (Escala D: Distimia). Además, en 1998, en la adaptación al castellano en España por Ávila-Espada, contó con 3 tipos de validez: de contenido, de criterio y de construcción por análisis factorial; así como con índices que oscilan entre .67 y .94 , en lo que refiere a la confiabilidad de consistencia interna. Asimismo, las escalas que ostentan mejor fiabilidad son los estilos Autoderrotista, Borderline, entre otros. Por otro lado, los coeficientes obtenidos en los estilos Esquizoide, Histriónico y Compulsivo son más bajos, pero aún aceptables (Millon \& Davis, 1998).

\section{Procedimiento}

Se tramitaron las autorizaciones de los establecimientos penitenciarios en la región Lima y de provincias, del Instituto Nacional Penitenciario (INPE). Después de recibir el permiso correspondiente, se procedió a la selección de las muestras en coordinación con nuestros asesores. Una vez obtenido el acceso a los pabellones determinados, se procedió a la aplicación de las pruebas de manera colectiva en pequeños grupos de 15 participantes, contando con la ayuda de los psicólogos responsables de cada pabellón; este proceso tuvo una duración promedio de seis meses, en el año 2017, en los turnos mañana y tarde, según las facilidades que se brindaron, tanto en Lima como en provincias. En el primer contacto con los participantes se les explicó de manera detallada el motivo de la aplicación de las pruebas para obtener el consentimiento informado de cada uno; sobre todo, se les hizo mención que los datos serían utilizados solamente con fines de investigación y que la participación sería voluntaria, incidiendo en la confidencialidad de sus 
identidades en la información que brinden. Asimismo, se explicaron las instrucciones para el llenado de las pruebas. Una vez obtenidos estos datos, se procedió a calificar e interpretar las pruebas administradas a los internos de los establecimientos penitenciarios, para luego realizar el análisis estadístico.

\section{Análisis de datos y confiabilidad de los instrumentos}

Una vez recopilados los instrumentos se procedió a registrar los datos en una matriz de recepción de datos, especialmente preparada en el programa estadístico SPSS versión 22.0. En dicha matriz, cada columna representaba un ítem o pregunta, y cada fila incorporaba las respuestas correspondientes a un encuestado. El proceso de datos se realizó sobre la base del análisis estadístico descriptivo y correlacional. Para el análisis descriptivo se utilizaron tablas de frecuencias, con el fin de realizar la exploración de cada una de las escalas del Inventario Clínico Multiaxial de Millon II y las dimensiones de la Escala de Asertividad Sexual (SAS). Para el análisis estadístico inferencial, primero se comprobó el supuesto de normalidad de las variables mediante la prueba Kolmogórov-Smirnov, y se encontró que no todas las variables estudiadas presentan distribución normal, por lo cual, se utilizó el coeficiente rho de Spearman para la prueba de hipótesis.

En cuanto a la confiabilidad y validez de los instrumentos utilizados en la muestra investigada, la fiabilidad global de la Escala de Asertividad Sexual y sus dimensiones se estimó calculando el índice de la consistencia interna mediante el coeficiente alpha de Cronbach (Tabla 3). Se aprecia que la consistencia interna global de la escala (18 ítems) en la muestra estudiada es de .828 , que puede ser considerada como indicador de una elevada fiabilidad. Además, se aprecia que la mayoría de las dimensiones presentan adecuados niveles de fiabilidad.

En cuanto a la validez de constructo del SAS, los coeficientes de correlación Producto-Momento de Pearson ( $r$ ) son significativos, confirmando así la validez de constructo que presenta la escala. De esa misma manera, los coeficientes que resultan de la correlación entre cada una de las dimensiones y el constructo en su globalidad son moderados en su mayoría, además de ser altamente significativos. 
Respecto al Inventario Clínico Multiaxial de Millon (MCMI-II), la fiabilidad de la escala se valoró calculando el índice de consistencia interna mediante el coeficiente alpha de Cronbach. En la Tabla 4 se observa que las consistencias internas del inventario en la muestra estudiada en su mayoría son adecuadas.

En el análisis de la validez del MCMI-II, los coeficientes de correlación Producto-Momento de Pearson $(r)$ son significativos entre los diferentes componentes de la escala, evidenciando que existe validez de constructo de la escala.

Tabla 3

Estimaciones de consistencia interna de la escala de Asertividad sexual

\begin{tabular}{lcc}
\hline & No $^{0}$ de ítems & Alpha \\
\hline Asertividad sexual global & 18 & .828 \\
Inicio de la actividad sexual & 6 & .745 \\
Rechazo de la actividad sexual & 6 & .714 \\
Prevención de ETS & 6 & .642 \\
\hline
\end{tabular}

Nota: Elaboración propia.

Tabla 4

Estimaciones de consistencia interna del Inventario Clínico Multiaxial de Millon II

\begin{tabular}{lcc}
\hline & $N^{0}$ de ítems & Alpha \\
\hline Esquizoide & 35 & .565 \\
Evitativo & 39 & .802 \\
Dependiente & 36 & .403 \\
Histriónico & 40 & .565 \\
Narcisista & 48 & .596 \\
Antisocial & 45 & .729 \\
Agresivo-sádico & 44 & .627 \\
Compulsivo & 38 & .607 \\
Pasivo-agresivo & 40 & .784 \\
Autoderrotista & 40 & .809 \\
Esquizotípico & 44 & .816 \\
Borderline & 60 & .893 \\
Paranoide & 44 & .765 \\
\hline
\end{tabular}

Nota: Elaboración propia. 


\section{Resultados}

En la Tabla 5 los patrones clínicos de personalidad que se encuentran en mayor número en los internos evaluados, son la personalidad compulsiva $(51.6 \%)$, dependiente $(50.5 \%)$, esquizoide $(44.6 \%)$, evitativa $(40.8 \%)$ y autoderrotista $(37 \%)$. Además, se aprecia que muy pocos internos presentan un estilo de personalidad histriónico $(7.6 \%)$.

\section{Tabla 5}

Patrones clínicos de personalidad

\begin{tabular}{lrccrcr}
\hline & \multicolumn{2}{c}{ Leve } & \multicolumn{2}{c}{ Moderado } & \multicolumn{2}{c}{ Severo } \\
\cline { 2 - 7 } & \multicolumn{1}{c}{$n$} & $\%$ & $n$ & $\%$ & $n$ & \multicolumn{1}{c}{$\%$} \\
\hline Esquizoide & 67 & $36.4 \%$ & 35 & $19 \%$ & 82 & $44.6 \%$ \\
Evitativo & 66 & $35.9 \%$ & 43 & $23.4 \%$ & 75 & $40.8 \%$ \\
Dependiente & 47 & $25.5 \%$ & 44 & $23.9 \%$ & 93 & $50.5 \%$ \\
Histriónico & 137 & $74.5 \%$ & 33 & $17.9 \%$ & 14 & $7.6 \%$ \\
Narcisista & 91 & $49.5 \%$ & 34 & $18.5 \%$ & 59 & $32.1 \%$ \\
Antisocial & 97 & $52.7 \%$ & 35 & $19 \%$ & 52 & $28.3 \%$ \\
Agresivo-sádico & 90 & $48.9 \%$ & 40 & $21.7 \%$ & 54 & $29.3 \%$ \\
Compulsivo & 48 & $26.1 \%$ & 41 & $22.3 \%$ & 95 & $51.6 \%$ \\
Pasivo-agresivo & 111 & $60.3 \%$ & 21 & $11.4 \%$ & 52 & $28.3 \%$ \\
Autoderrotista & 64 & $34.8 \%$ & 52 & $28.3 \%$ & 68 & $37 \%$ \\
\hline
\end{tabular}

Nota: Elaboración propia.

En la Tabla 6 se aprecia que $23.4 \%$ de los internos presentan indicadores severos de patrones de personalidad esquizotípico, y $20.7 \%$ presentan indicadores severos de patrones de personalidad paranoide. Además, solo $14.7 \%$ de los internos presentan indicadores severos de personalidad borderline. 
Tabla 6

Patrones severos de la personalidad

\begin{tabular}{lrrrrrr}
\hline & \multicolumn{2}{c}{ Leve } & \multicolumn{2}{c}{ Moderado } & \multicolumn{2}{c}{ Severo } \\
\cline { 2 - 7 } & \multicolumn{1}{c}{$n$} & \multicolumn{1}{c}{$\%$} & $n$ & \multicolumn{1}{c}{$\%$} & \multicolumn{1}{c}{$n$} & \multicolumn{1}{c}{$\%$} \\
\hline Esquizotípico & 123 & $66.8 \%$ & 18 & $9.8 \%$ & 43 & $23.4 \%$ \\
Borderline & 127 & $69 \%$ & 30 & $16.3 \%$ & 27 & $14.7 \%$ \\
Paranoide & 124 & $67.4 \%$ & 22 & $12 \%$ & 38 & $20.7 \%$ \\
\hline
\end{tabular}

Nota: Elaboración propia.

En la Tabla 7 se observa que solo $22.3 \%$ de los internos presentan un nivel alto de asertividad sexual, y la mayoría presentan nivel entre bajo y moderado de asertividad sexual. De manera similar ocurre con las dimensiones de la asertividad sexual, donde solo $16.3 \%$ tienen nivel alto de asertividad en el inicio de la actividad sexual. Asimismo, 26.6\% presentan nivel alto de asertividad al rechazar la actividad sexual y $28.3 \%$ presentan nivel alto de asertividad en la prevención de enfermedades de transmisión sexual.

\section{Tabla 7}

Nivel de asertividad sexual

\begin{tabular}{lcccccc}
\hline & \multicolumn{2}{c}{ Bajo } & \multicolumn{2}{c}{ Moderado } & \multicolumn{2}{c}{ Alto } \\
\cline { 2 - 7 } & $n$ & $\%$ & $n$ & $\%$ & $n$ & $\%$ \\
\hline $\begin{array}{l}\text { Asertividad sexual } \\
\text { Inicio de la actividad }\end{array}$ & 48 & $26.1 \%$ & 95 & $51.6 \%$ & 41 & $22.3 \%$ \\
$\begin{array}{l}\text { sexual } \\
\text { Rechazo de la actividad }\end{array}$ & 54 & $29.3 \%$ & 100 & $54.3 \%$ & 30 & $16.3 \%$ \\
$\begin{array}{l}\text { sexual } \\
\text { Prevención de ETS }\end{array}$ & 42 & $22.8 \%$ & 93 & $50.5 \%$ & 49 & $26.6 \%$ \\
\hline
\end{tabular}

Nota: Elaboración propia.

\section{Prueba de normalidad}

Con el propósito de contrastar las hipótesis planteadas, se procedido a realizar la prueba de bondad de ajuste para evaluar si las variables presentaban una distribución normal. En la Tabla 8 se presentan los resultados de la prueba de bondad de ajuste de Kolmogorov-Smirnov (KS) donde se observa que los datos correspondientes a las 17 variables, en 7 
de ellas no presentan distribución normal dado que el coeficiente obtenido $(\mathrm{K}-\mathrm{S})$ es significativo $(p<.05)$. Por lo cual se utilizó el estadístico no paramétrico para la prueba de las hipótesis.

\section{Tabla 8}

Prueba de bondad de ajuste a la curva normal para las variables en estudio

\begin{tabular}{llcccc}
\hline \multirow{1}{*}{ Instrumentos } & Variables & Media & $D E$ & K-S & P \\
\hline & Esquizoide & 28.4 & 7.68 & .048 & .200 \\
& Evitativo & 27.1 & 11.6 & .057 & .200 \\
& Dependiente & 35.5 & 6.42 & .068 & .037 \\
& Histriónico & 32.9 & 8.17 & .058 & .200 \\
& Narcisista & 37.9 & 9.22 & .047 & .200 \\
Patrones clínicos & Antisocial & 33.1 & 11.7 & .070 & .029 \\
de personalidad & Agresivo-sádico & 32.8 & 9.21 & .044 & .200 \\
& Compulsivo & 45.1 & 8.15 & .092 & .001 \\
& Pasivo-agresivo & 28.2 & 12.3 & .064 & .064 \\
& Autoderrotista & 28.5 & 12.0 & .054 & .200 \\
& Esquizotípico & 30.8 & 12.9 & .051 & .200 \\
& Borderline & 31.4 & 17.8 & .062 & .077 \\
& Paranoide & 38.4 & 11.3 & .047 & .200 \\
Asertividad sexual & Puntaje global & 36.1 & 6.57 & .106 & .000 \\
& Inicio & 11.5 & 2.27 & .122 & .000 \\
& Rechazo & 12.5 & 3.46 & .176 & .000 \\
& Prevención de ETS & 12.1 & 4.33 & .142 & .000 \\
\hline
\end{tabular}

Nota: Elaboración propia.

\section{Discusión}

Actualmente, la asertividad sexual juega un rol muy importante en la sexualidad humana, especialmente en la salud sexual (Santos-Iglesias \& Sierra, 2010), pues se constituye en una habilidad social que permite a la persona comunicar de manera adecuada necesidades y preferencias sexuales a su pareja. Frente a ello, los patrones de personalidad están relacionados de manera negativa con esta capacidad, como se aprecia al contrastar las hipótesis de este estudio (ver Tabla 9). De los 13 tipos de personalidad, 11 presentan una relación significativa y negativa con el nivel de asertividad sexual en los internos de cuatro centros penitenciarios de Perú. 
Tabla 9

Coeficientes de correlación entre patrones clínicos de personalidad y asertividad sexual

\begin{tabular}{lcccccccc}
\hline & \multicolumn{3}{c}{ Asertividad sexual } & \multicolumn{2}{c}{ Inicio } & \multicolumn{2}{c}{ Rechazo } & \multicolumn{2}{c}{ Prevención de ETS } \\
\cline { 2 - 9 } & rho & $p$ & rho & $p$ & rho & $p$ & rho & $p$ \\
\hline Esquizoide & -.221 & .003 & .016 & .828 & -.221 & .003 & -.224 & .002 \\
Evitativo & -.234 & .001 & -.015 & .843 & -.237 & .001 & -.151 & .041 \\
Dependiente & -.176 & .017 & -.215 & .003 & -.078 & .290 & -.085 & .251 \\
Histriónico & -.058 & .436 & -.252 & .001 & -.066 & .371 & .103 & .163 \\
Narcisista & -.157 & .034 & -.110 & .139 & -.131 & .077 & -.062 & .401 \\
Antisocial & -.160 & .030 & -.072 & .331 & -.141 & .057 & -.083 & .263 \\
Agresivo-sádico & -.171 & .021 & -.114 & .123 & -.162 & .028 & -.076 & .303 \\
Compulsivo & -.108 & .143 & -.162 & .028 & -.046 & .535 & -.092 & .214 \\
Pasivo-agresivo & -.166 & .025 & -.071 & .341 & -.156 & .034 & -.082 & .270 \\
Autoderrotista & -.264 & .000 & -.141 & .056 & -.214 & .004 & -.119 & .108 \\
Esquizotípico & -.224 & .002 & -.054 & .467 & -.213 & .004 & -.123 & .098 \\
Borderline & -.252 & .001 & -.101 & .173 & -.198 & .007 & -.131 & .077 \\
Paranoide & -.204 & .005 & -.143 & .053 & -.128 & .084 & -.163 & .027 \\
\hline
\end{tabular}

Nota: Elaboración propia.

Siguiendo la clasificación dada por el Manual de Diagnóstico y Estadístico de los Trastornos Mentales (Asociación Americana de Psiquiatría, 2014), en el grupo A, el patrón de personalidad paranoide presenta una relación altamente significativa y negativa con la asertividad sexual ( $r h o=-.204, p<.01$ ), es decir, una mayor presencia y gravedad de rasgos disfuncionales de la personalidad paranoide está relacionada con un menor nivel de asertividad sexual. Esta personalidad tiene muchas dificultades en su interacción con los demás, especialmente al expresar sus opiniones, ya que presenta un patrón marcado de desconfianza y suspicacia frente a otros (Millon et al., 2006). Estos patrones hacen evidente su relación negativa con la asertividad sexual, ya que tienen muchas dificultades para expresar sus deseos sexuales o están siempre predispuestos para a contraatacar con rapidez y reaccionar con ira ante la mínima provocación percibida por ellos. También el patrón de personalidad esquizoide presenta una relación altamente significativa y negativa ( $r h o=$ $-.221, p<.01$ ) con la asertividad sexual. Esta relación se hace evidente ya que este patrón de personalidad se distingue porque el sujeto presenta un marcado distanciamiento en la interacción con sus pares, no hay un deseo de 
intimidad, tampoco un deseo de formar una familia o grupo social y muestra un interés disminuido por tener relaciones sexuales con otra persona (Millon, 1999). De manera similar, el patrón de personalidad esquizotípico presenta una relación significativa y negativa ( $r h o=-.224, p<.01)$ debido a que una característica esencial de este tipo es la incapacidad para utilizar todo la gama de afectos y habilidades para interactuar de manera adecuada y efectiva con los demás, lo que hace que tengan un comportamiento inapropiado e inflexible (Asociación Americana de Psiquiatría, 2014; Caballo, Salazar, \& Carrobles, 2014). Estos patrones evidencian que la persona tiene muchas dificultades para iniciar la actividad sexual, rechazar la actividad sexual no deseada y negociar el uso de métodos anticonceptivos de barrera.

Con respecto al grupo B, el patrón antisocial de la personalidad presenta una relación significativa y negativa con el nivel de asertividad sexual ( $r$ ho $=$ -.160, $p<.05)$, es decir, una mayor presencia y gravedad de rasgos disfuncionales de la personalidad antisocial está relacionado con un menor nivel de asertividad sexual en los internos. Esta relación se hace evidente ya que esta personalidad se caracteriza por una carencia de empatía y la ausencia de sensibilidad frente a los sentimientos y derechos de los demás. También se caracterizan por ser irresponsables, impulsivos y manipuladores en sus relaciones sexuales además de tener múltiples parejas sexuales (Asociación Americana de Psiquiatría, 2014; Millon, 2006). El patrón de personalidad límite también presenta una relación altamente significativa y negativa con el nivel de asertividad sexual ( $r h o=-.252, p<.01$ ). Esto debido a su marcada inestabilidad emocional e impulsividad, donde pueden involucrarse en prácticas sexuales de riesgo (Millon, Grossman, Millon, Meagher, \& Ramnath, 2006). Además, el patrón de personalidad narcisista presenta una relación significativa y negativa con el nivel de asertividad sexual $(r h o=-.157, p<.05)$, esto debido a que tienen un sentido grandioso de su persona, presentan escasa empatía y se les dificulta reconocer los deseos y sentimientos de los demás (Asociación Americana de Psiquiatría, 2014). Sin embargo, el patrón de personalidad histriónico no presenta una relación significativa con el nivel de asertividad sexual $(r h o=-.058, p>.05)$. Si bien este tipo de personalidad se caracteriza por tener un comportamiento provocador y seductor hacia la otra persona además de una inestabilidad emocional, esto no guarda relación con la asertividad sexual. Esto podría deberse a que los participantes son varones y hay muy pocos que presentan este tipo. 
En el grupo C, el patrón de personalidad por evitación presenta una relación altamente significativa y negativa con el nivel de asertividad sexual $(r h o=-.234, p<.01)$. Debido a que destaca en esta personalidad la inhibición social, el sentirse inadecuado y una percepción exagerada de estar siendo evaluado todo el tiempo. Esto hace que iniciar una actividad sexual o rechazar una actividad sexual de manera asertiva les resulte muy difícil (Asociación Americana de Psiquiatría, 2014). El patrón de personalidad por dependencia presenta una relación significativa y negativa con el nivel de asertividad sexual $(r h o=-.176, p<.05)$. Se debe a que esta personalidad se caracteriza por una necesidad general y excesiva de dependencia de alguien que ocasiona un comportamiento sumiso y con temor al abandono. Además, la dificultad para expresar sus emociones y opiniones, así como mostrar su desacuerdo (Asociación Americana de Psiquiatría, 2014) hace que tengan muchas dificultades para iniciar, rechazar o negociar el uso de métodos anticonceptivos en la actividad sexual. Por otro lado, el patrón obsesivocompulsivo no presenta una relación significativa con el nivel de asertividad sexual ( $r h o=-.108, p>.05)$, pero sí con uno de sus componentes, con el inicio de la actividad sexual $(r h o=-.162, p<.05)$. Esto debido a que la característica esencial de este trastorno es una preocupación por el orden, el perfeccionismo y el control estricto de su comportamiento (Millon et al., 2006), lo que hace que tengan dificultad para iniciar una actividad sexual.

Por otro lado, se encontró una relación significativa entre el patrón de personalidad agresivo-sádico y el nivel de asertividad sexual ( $r h o=-.171, p<$ .05). Este patrón de personalidad encuentra placer en humillar, intimidar y menospreciar a otras personas (Millon et al., 2006), todo ello hace que tenga muchas dificultades en la actividad sexual. El patrón de personalidad pasivoagresivo presenta una relación significativa y negativa con el nivel de asertividad sexual ( $r h o=-.166, p<.05)$. Obedece a que este patrón de personalidad está predispuesto a la hostilidad, a discutir y una ambivalencia en su conducta (Millon et al., 2006).

Finalmente, el patrón de personalidad autoderrotista presenta una relación altamente significativa y negativa con el nivel de asertividad sexual $(r h o=$ -.264, $p<.05$ ). Esta personalidad se caracteriza por evitar o socavar las experiencias placenteras, se involucra en relaciones perjudiciales que le producen frustración, fracaso e incluso a ser maltratado y rechazar a personas 
que le traten con respeto (Millon et al., 2006). Todo ello hace muy difícil para este tipo de personas tener asertividad sexual en la intimidad.

\section{Contraste con la teoría y resultados de otras investigaciones}

Con respecto a los patrones de personalidad y la asertividad sexual, están relacionados de manera negativa con dicha capacidad, como se muestra al contrastar las hipótesis del presente estudio. Así, de los 13 patrones de personalidad, 11 de ellos presentan una relación significativa y negativa con el nivel de asertividad sexual en los internos de cuatro centros penitenciarios del país. Este resultado coincide con los estudios de Firoozi, Asmoude y Asgharipoor (2016); Miri, AliBesharat, Asadi y Shahyad (2011) y Moyano y Sierra (2013), donde algunos de los patrones de personalidad influyen significativamente sobre la sexualidad de la persona.

Tomando en consideración la clasificación dada por el Manual de diagnóstico y estadístico de los trastornos mentales (Asociación Americana de Psiquiatría, 2014); con respecto al grupo B, el patrón antisocial de la personalidad presenta una relación significativa y negativa con el nivel de asertividad sexual ( $r h o=-.160, p<.05)$, es decir, una mayor presencia y gravedad de rasgos disfuncionales de la personalidad antisocial está relacionado con un menor nivel de asertividad sexual en los internos. Al comparar con otras investigaciones relacionadas con el objetivo del estudio, se encontró que concuerda con lo reportado por Ortiz-Tallo, Sánchez y Cardenal (2002) y por Castro, López y Sueiro (2009). Además, esta relación se hace evidente ya que esta personalidad se caracteriza por una carencia de empatía e insensibilidad frente a los sentimientos y derechos de los demás. También se caracterizan por ser irresponsables, impulsivos y manipuladores en sus relaciones sexuales además de tener múltiples parejas sexuales (Asociación Americana de Psiquiatría, 2014; Millon et al., 2006). El patrón de personalidad limite, asimismo, presenta una relación altamente significativa y negativa con el nivel de asertividad sexual $(r h o=-.252, p<.01)$. Esto coincide con el estudio de Castro et al. (2009), donde los rasgos de personalidad límite están presentes en los internos condenados por delito sexual. Lo que sucede, probablemente a su marcada inestabilidad emocional e impulsividad, ya que pueden involucrarse en prácticas sexuales de riesgo (Millon et al., 2006). 
Por otro lado, se encontró una relación significativa entre el patrón de personalidad agresivo-sádico y el nivel de asertividad sexual ( $r h o=-.171, p<$ .05), el cual coincide con los resultados encontrados por Castro et al. (2009). Este tipo de personalidad encuentra placer en humillar, intimidar y menospreciar a otras personas (Millon et al., 2006), todo ello hace que tenga muchas dificultades en la actividad sexual.

Respecto a las variables investigadas, no se encontraron otras investigaciones que tengan una relación directa entre patrones de personalidad y la asertividad sexual; las halladas, establecen una relación por separado, tanto con la variable personalidad, como con la variable asertividad sexual.

\section{Conclusiones}

No se encuentra un patrón de características de personalidad o perfil definido en el agresor sexual, ya que, de los 13 patrones de personalidad, 11 de ellos presentan una relación significativa y negativa con el nivel de asertividad sexual; siendo este bajo en los internos de cuatro centros penitenciarios del país.

Se halló dos personalidades que no presentan relación significativa con el nivel de asertividad sexual de los reclusos evaluados: la personalidad histriónica y la compulsiva. Los demás patrones de personalidad sí guardan relación significativa con el nivel de asertividad sexual.

Respecto a las dimensiones de asertividad sexual, la personalidad esquizoide, antisocial, esquizotípica, no presentan relación significativa con el inicio de la actividad sexual. Los demás patrones de personalidad sí guardan relación significativa con esta variable. Asimismo, la personalidad dependiente, histriónica, narcisista, antisocial, compulsiva y paranoide no presentan relación significativa con el rechazo de la actividad sexual. Los demás patrones de personalidad sí guardan relación significativa con esta variable. Finalmente, solo las personalidades esquizoide, evitativa y paranoide, presentan relación significativa con la prevención de enfermedades de transmisión sexual. Los demás patrones de personalidad no presentan relación significativa con esta variable. 


\section{Referencias}

Asociación Americana de Psiquiatría. (2014). Manual diagnóstico y estadístico de los trastornos mentales, DSM5 (5a ed.). Madrid: Panamericana.

Caballo, V., Salazar, I., \& Carrobles, J. (2014). Manual de psicopatología y trastornos psicológicos (2a ed.). Madrid: Pirámide.

Castro, M. E., López, A., \& Sueiro, E. (2009). Perfil psicopatológico de agresores sexuales. Cuaderno de Medicina Psicosomática y Psiquiatría de Enlace, 89(90), 30-39. Recuperado de http://www.editorialmedica.com/ download.php?idart=339

De la Torre, B. (2012). Personalidad y agresión sexual (Tesis de maestría). Universidad Autónoma de Madrid. Recuperado de http://portal.uned.es/pls/portal/docs/PAGE/UNED_MAIN/LAUNIVERSIDAD/VICE RRECTORADOS/GERENCIA/IUISI/COLABORACIONES/078\%20DOC_ISIE_10_2012.PDF

Firoozi, M., Asmoude, E., \& Asgharipoor, N. (2016). The relationship between personality traits and sexual selfesteem and its components. Iranian Jounal of Nursing and Midwifery Research, 21(3), 225-231. Recuperado de https://www.ncbi.nlm.nih.gov/pmc/articles/PMC4857655/

Jiménez, P. (2009). Caracterización psicológica de un grupo de delincuentes sexuales chilenos a través del Test de Rorschach. Psykhe, 18(1), 27-38. Recuperado de http://www.scielo.cl/pdf/psykhe/v18n1/art03.pdf

Larrotta, R., \& Rangel-Noriega, K. (2013). El agresor sexual. Aproximaciones teóricas a su caracterización. Informes Psicológicos, 13(2), 103-120. Recuperado de https://revistas.upb.edu.co/index.php/ informespsicologicos/article/view/2795/2440

Millon, T. (1999). Manual del Inventario Clínico Multiaxial de Millon, MCMI-II (2ª ed.). Madrid: TEA Ediciones.

Millon, T., \& Davis, R. (1998). Trastornos de la personalidad. Más allá del DSM-IV. Barcelona: Masson.

Millon, T., Grossman, S., Millon, C., Meagher, S., \& Ramnath, R. (2006). Trastornos de la personalidad en la vida moderna ( $2^{\mathrm{a}}$ ed.). Barcelona: Masson.

Miri, M., AliBesharat, M., Asadi, M., \& Shahyad, S. (2011). The relationship between dimensions of personality and sexual desire in females and males. Procedia Social and Behavioral Sciences, 15, 823-827. Recuperado de https://www.sciencedirect.com/science/article/pii/S1877042811003715

Moyano, N., \& Sierra, J. C. (2013). Relationships between Personality Traits and Positive/Negative Sexual Cognitions. International Jjournal of clinical and health psychology, 13(3), 189-196. Recuperado de https:/l www.sciencedirect.com/science/article/pii/S1697260013700231

Organización Panamericana de la Salud. (2013). Comprender y abordar la violencia contra las mujeres. Violencia sexual. Recuperado de https://www.who.int/reproductivehealth/publications/violence/rhr12_37/es/

Ortiz-Tallo, M., Sánchez, L., \& Cardenal, V. (2002). Perfil psicológico de delincuentes sexuales. Un estudio clínico con el MCMI-II de Th. Millon. Revista de Psiquiatría, 29(3), 144-153. Recuperado de https:/l www.uma.es/Psicologia/docs/eudemon/divulgacion/perfil_psicologico_delincuentes_sexuales.pdf

Ramírez, W. (2002). Caracterización de ofensores sexuales juveniles: Experiencia de la clínica de adolescentes del Hospital Nacional de Niños. Acta Pediátrica Costarricense, 16(2), 69-74. Recuperado de http:/l www.scielo.sa.cr/scielo.php ?script=sci_arttext\&pid=S1409-00902002000200005

Sánchez, H., Reyes, C., \& Mejía, K. (2015). Manual de términos en investigación científica, tecnológica y humanística. Recuperado de http://repositorio.urp.edu.pe/bitstream/handle/URP/1480/libro-manual-determinos-en-investigacion.pdf?sequence=1\&isAllowed=y

Santos-Iglesias, P., \& Sierra, J. C. (2010). El papel de la asertividad sexual en la sexualidad humana: Una revisión sistemática. International journal of clinical and health psychology, 10(3), 553-577. 
Sierra, J. C., Vallejo-Medina, P., \& Santos-Iglesias, P. (2011). Propiedades psicométricas de la versión española de la Sexual Assertiveness Scale (SAS). Anales de Psicología, 27(1), 17-26. Recuperado de http:/l revistas.um.es/analesps/article/view/113431/107421

Soria, M., \& Hernández, J. (1994). El agresor sexual y la víctima. Barcelona: Marcombo.

Torres-Obregón, R., Onofre-Rodríguez, D., Sierra, J. C., Benavides-Torres, R., \& Garza-Elizondo, M. E. (2017). Validación de la sexual assertiveness scale en mujeres mexicanas. Suma Psicológica, 24, 34-41. Recuperado de http://www.scielo.org.co/pdf/sumps/v24n1/0121-4381-sumps-24-01-00034.pdf

Vásquez, B. (2005). Manual de psicología forense. Madrid: Síntesis.

Zerubavel, N., \& Messman-Moore, T. (2013). Sexual victimization, fear of sexual powerlessness, and cognitive emotion dysregulation as barriers to sexual assertiveness in college women. Violence Against Women, 19(12), 1518-1537. Recuperado de https://www.ncbi.nlm.nih.gov/pubmed/24379216 